\title{
CHROMOBACTERIUM VIOLACEUM WOUND INFECTION
}

\author{
Atanu Chakravarty ${ }^{1}$, Anuradha Modak ${ }^{2}$
}

${ }_{1}^{1}$ Assistant Professor, Department of Microbiology, Silchar Medical College and Hospital, Assam, India.

2Postgraduate Trainee, Department of Microbiology, Silchar Medical College and Hospital, Assam, India.

HOW TO CITE THIS ARTICLE: Chakravarty A, Modak A. Chromobacterium violaceum wound infection. J. Evolution Med. Dent. Sci. 2018;7(31):3560-3562, DOI: 10.14260/jemds/2018/799

\section{PRESENTATION OF CASE}

A 45 years old female from Mizoram, visited the Orthopaedics OPD of SMCH on 24th January 2017 with complaints of pain, swelling and discharge from the fractured wound for last 6 months. She already had an implant on her fractured right leg, operated at the Civil Hospital, Mizoram. She got this fracture injury around 6 months back in a football ground, while accompanying her son. She lived in an area with no mud flows or landslides and did not report using the groundwater or contaminated water. She is non-diabetic, non-hypertensive, no suggestive past history or family history. She is a chronic smoker.

On admission, she was febrile with respiratory rate of 22 breaths/min, heart rate of 110 beats/min and blood pressure of $110 / 70 \mathrm{mmHg}$. She was conscious and alert. No suggestive findings in central nervous system, cardiovascular system, respiratory system or gastrointestinal system. On local examination, she had fracture wound both bones of right leg with profuse discharge and oedema of that region [Figure 1].

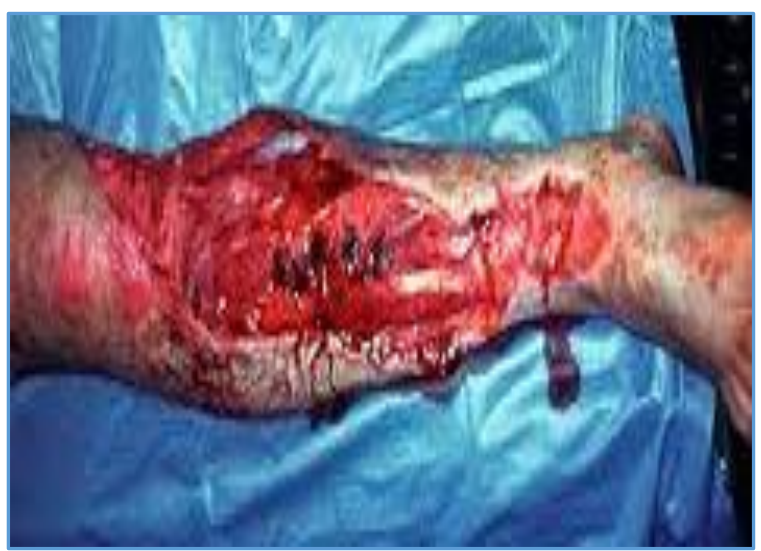

Figure 1. Wound Infection of Right Leg with Both Bone Fracture

She was planned for implant removal, wound debridement with antibiotic beads and external fixator application. Discharge from that wound was collected in a sterile swab stick and sent for culture and sensitivity examination. Patient was gradually improving post operation. She was responding to the Aminoglycoside antibiotic treatment. History and all investigations were taken after informed consent of the patient.

'Financial or Other Competing Interest': None.

Submission 18-06-2018, Peer Review 11-07-2018,

Acceptance 19-07-2018, Published 30-07-2018.

Corresponding Author:

Dr. Anuradha Modak

Department of Microbiology,

Silchar Medical College and Hospital,

Assam, India.

E-mail: anuradhamodak98@gmail.com

DOI: $10.14260 /$ jemds/2018/799

\section{CLINICAL DIAGNOSIS}

Pseudomonas (pigmented strain) infection.

The classical history and non-response to the commonly used broad-spectrum antibiotics in our hospital indicated that the infecting organism is resistant to antibiotics and not usually encountered in our hospital. So, pus sample of the wound was also sent for microbiological examination.

\section{PATHOLOGICAL DISCUSSION}

The Gram stain of direct smear of the pus sample showed plenty of pus cells along with Gram-negative rods. The other portion of the sample was inoculated on Nutrient, Blood and MacConkey agar media and incubated aerobically at $37^{\circ} \mathrm{C}$ for 24 hours. On next day the type of growth was smooth, round, convex, butyrous and violet-coloured on all three plates. On Blood agar, colonies were deep-violet and with betahaemolysis [Figures- 2 and 3]. The organism was processed from culture growth and it was facultative anaerobic, motile and Gram-negative rod. It was catalase and oxidase positive. Indole, Methyl-red and Voges-Proskauer tests showed negative results. The organism fermented glucose (producing acid but no gas) and trehalose, but not lactose or mannitol. Triple sugar iron medium was with alkaline slant and acid butt (K/A) without gas and $\mathrm{H}_{2} \mathrm{~S}$ production. Citrate was utilised, and nitrate was reduced to nitrite. Arginine was dihydrolysed but lysine and ornithine were not. A second pus sample was collected on the following day of first sample and the same organism was isolated again, which proved that the organism was a true pathogen and not a contaminant. Phenotypically, this isolate was identified as Chromobacterium violaceum. ${ }^{3}$

Chromobacterium violaceum is a Gram-negative, facultative anaerobe, motile, oxidase and catalase positive bacilli which produce a purple pigment violacein which is alcohol-soluble, water and chloroform insoluble. It grows easily on Nutrient agar, Blood agar and MacConkey agar media with incubation at $30^{\circ} \mathrm{C}$ to $45^{\circ} \mathrm{C}$, producing distinctive smooth low convex colonies with a dark violet metallic sheen. It can be isolated from natural habitats in tropical and subtropical regions. The organism is unable to survive below $4^{\circ} \mathrm{C}$ and thrives at a temperature of $20^{\circ} \mathrm{C}$ to $37^{\circ} \mathrm{C}$. It is found in soil and water. ${ }^{2}$

Chromobacterium violaceum is a motile Gram-negative bacillus, a rare entity that usually starts with a localised skin infection or lymphadenitis after contact with contaminated water or soil. In humans it causes a rare tropical and subtropical disease. The awareness of this organism is limited in spite of its ubiquitous distribution. Here, we report a case of a middle-aged female with a history of fracture injury in a muddy field with subsequent infection of that site. 1 
Human infections caused by C. violaceum are rare. So to prevent serious infection in healthy people, rapid diagnosis, accurate bacterial identification and specific treatment is very important.

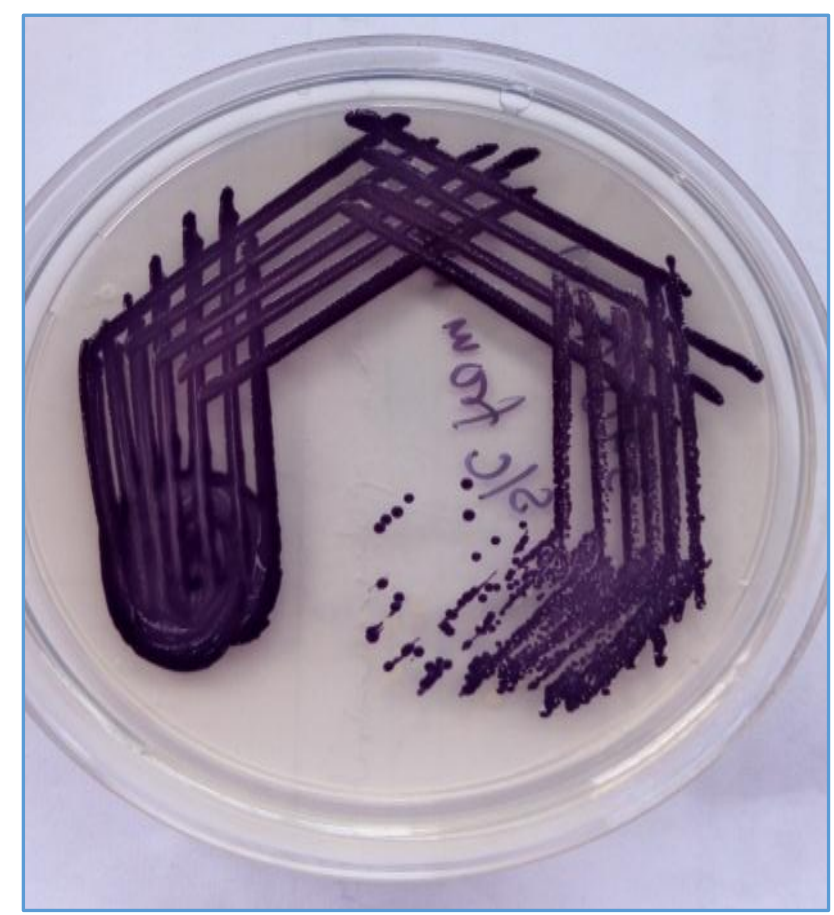

Figure 2. C. violaceum on Nutrient agar

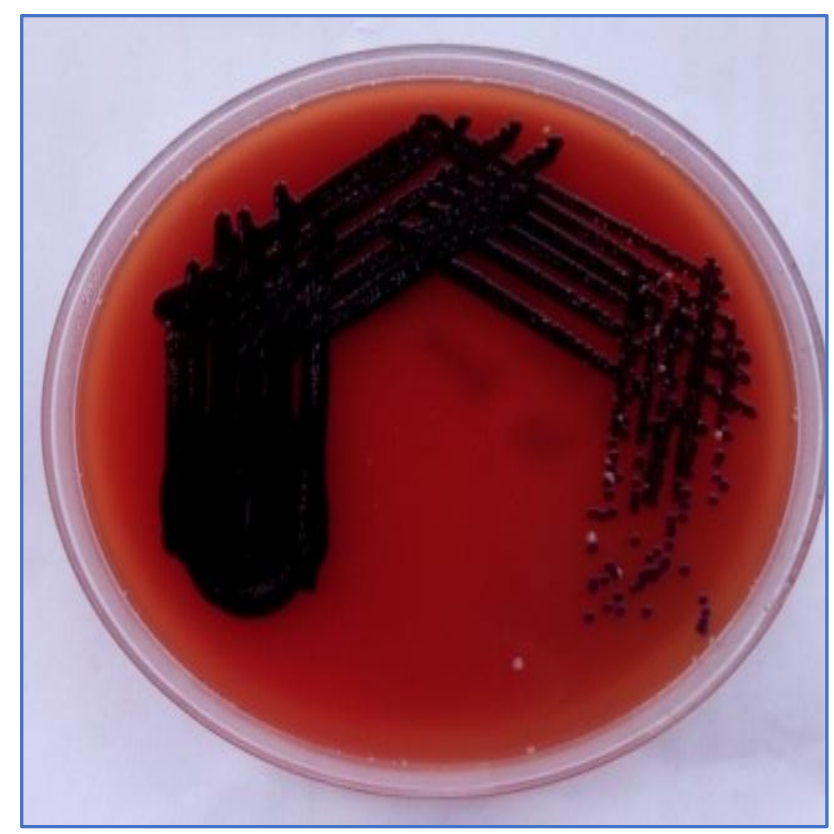

Figure 3. C. violaceum on Blood agar

\section{DISCUSSION OF MANAGEMENT}

Antibiotic sensitivity of the organism was done by the disc diffusion method. The organism was sensitive to Gentamicin, Ciprofloxacin, Imipenem and Amikacin [Figure 4]. It was intermediate sensitive to Cefotaxime, but was resistant to Ampicillin. Based on the antibiotic sensitivity result, the patient was treated with injection Gentamicin $80 \mathrm{mg}$ IV twice a day for 7 days. The wound healed completely after the treatment. The patient was followed up for next 15 days and the wound was completely healed with no other complications.

Most C. violaceum infections occur during the months of June through September. The organism enters the human body through minor trauma of the skin or ingestion of contaminated water. The main complications of the cases with C. violaceum infection are multiple liver abscesses, diffuse pustular dermatitis and sepsis. A study by M Ravish Kumar et al showed untreated C. violaceum infection causing brain abscesses and diarrhoea. C. violaceum usually gives pigmented colonies, but some non-pigmented strains have also been reported and this makes diagnosis even more difficult. Another study by Chen Chang-Hua et al showed C. violaceum bacteraemia with a fulminant clinical course that started from a small local wound infection. ${ }^{4}$

The virulence of $\mathrm{C}$. violaceum is attributed partly to endotoxin and to inadequate host defence. The host defence against the organism depends on adequate levels of superoxide dismutase and catalase. This organism is usually sensitive to Chloramphenicol, Imipenem, Gentamicin, Cotrimoxazole and Ciprofloxacin, but it is generally resistant to Penicillins, Cephalosporins and Aztreonam. ${ }^{1}$

In our case, the following points favoured the diagnosis of C. violaceum: typical history of fall into a muddy field, isolation of the same organism from the repeat pus sample and prompt response to the treatment with Aminoglycosides.

Prompt intervention with administration of antibiotic to which the organism was sensitive helped our patient's recovery. The wound healed completely without any complication. ${ }^{5}$

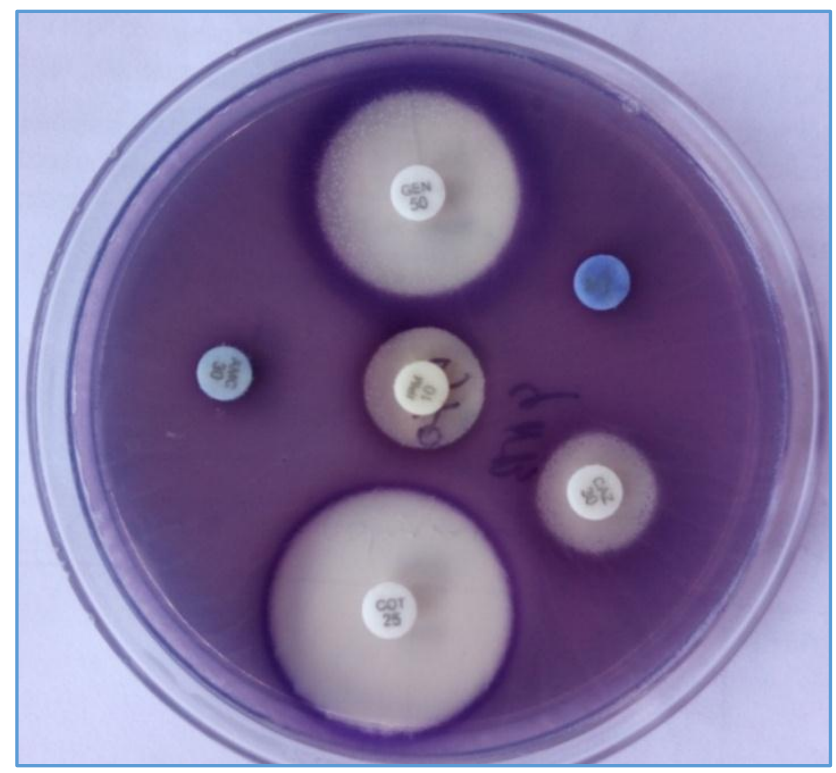

Figure 4. Antibiotic Sensitivity of C. violaceum from Nutrient agar Growth

On laboratory investigation, WBC count was $6400 / \mathrm{mm}^{3}$ with $61.6 \%$ neutrophils. $\mathrm{Hb} \%$ level was $12.7 \%$ and platelets were $3,40,000 / \mathrm{mm}^{3}$. Random blood sugar level was 85 $\mathrm{gm} / \mathrm{dL}$. Liver and renal function tests were within normal limits. 


\section{FINAL DIAGNOSIS}

C. violaceum Infection.

\section{REFERENCES}

[1] Kumar MR. Chromobacterium violaceum: a rare bacterium isolated from a wound over the scalp. International Journal of Applied and Basic Medical Research 2012;2(1):70-2.

[2] Yang $\mathrm{CH}, \mathrm{Li} \mathrm{YH}$. Chromobacterium violaceum infection: a clinical review of an important but neglected infection. Journal of the Chinese Medical Association 2011;74(10):435-41.
[3] Jitmuang A. Human Chromobacterium violaceum infection in Southeast Asia: case reports and literature review. Southeast Asian J Trop Med Public Health 2008;39(3):452-60.

[4] Moore CC, Lane JE, Stephens JL. Successful treatment of an infant with chromobacterium violaceum sepsis. Clinical Infectious Diseases 2001;32(6):E107-E10.

[5] Ray P, Sharma J, Marak RS, et al. Chromobacterium violaceum septicaemia from north India. Indian J Med Res 2004;120(6):523-6. 